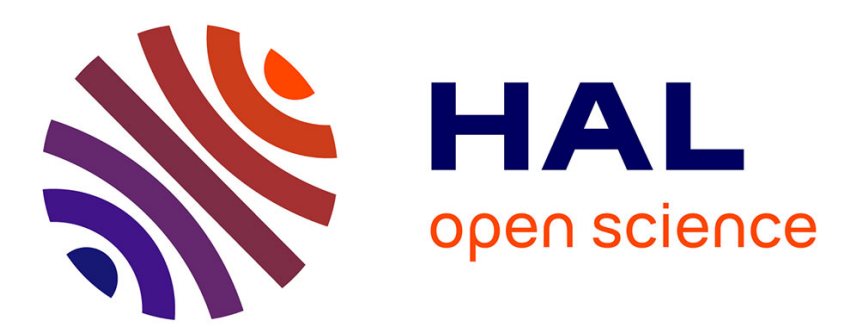

\title{
Characterisation of pendular capillary bridges derived from experimental data using inverse problem method
}

Boleslaw Mielniczuk, Olivier Millet, Gérard Gagneux, Moulay Saïd El Youssoufi

\section{- To cite this version:}

Boleslaw Mielniczuk, Olivier Millet, Gérard Gagneux, Moulay Saïd El Youssoufi. Characterisation of pendular capillary bridges derived from experimental data using inverse problem method. Granular Matter, 2018, 20 (1), pp.14. 10.1007/s10035-017-0784-8 . hal-01710311

\section{HAL Id: hal-01710311 \\ https://hal.science/hal-01710311}

Submitted on 15 Feb 2018

HAL is a multi-disciplinary open access archive for the deposit and dissemination of scientific research documents, whether they are published or not. The documents may come from teaching and research institutions in France or abroad, or from public or private research centers.
L'archive ouverte pluridisciplinaire HAL, est destinée au dépôt et à la diffusion de documents scientifiques de niveau recherche, publiés ou non, émanant des établissements d'enseignement et de recherche français ou étrangers, des laboratoires publics ou privés. 


\title{
Characterisation of pendular capillary bridges derived from experimental data using inverse problem method
}

\author{
B. Mielniczuk ${ }^{1,2,3} \cdot$ O. Millet $^{1}$ - G. Gagneux ${ }^{1}$ - M. S. El Youssoufi ${ }^{2,3}$
}

\begin{abstract}
In this study we use the recent analytical model to analyze capillary interactions in liquid bridge between two spherical grains, with fixed volumes of liquid and varying separation distance. Sequences of images of capillary bridges with different parameters are recorded during experimental tests. Geometrical parameters, as contact angle, half-filling angle and neck radius, are determined by image processing. Profiles of examined bridges are approximated as a Delaunay's roulette and superposed on recorded images. Evolution of associated variables (Laplace pressure, capillary force) is also calculated. Results of theoretical modeling are compared with the experimental ones. They match very accurately for small volumes and/or small separation distances, when influence of gravity is not significant. For larger liquid volumes and/or larger separation distances between grains the influence of the gravity is observed as a distortion (loss of symmetry) of capillary bridge. To avoid this deformation, several test were realized in micro-gravity conditions. For these tests, theoretical results are in good agreement with experimental ones, also for higher liquid volumes and/or separations distances.
\end{abstract}

Keywords Capillary bridge $\cdot$ Young-Laplace equation $\cdot$ Inverse problem $\cdot$ Experimental measurement

\section{Introduction}

Liquid links between solid grains give origin of several phenomena observed in unsaturated granular materials (soils, powders). They have significant influence on mechanical properties of these materials, because of intergranular capillary forces arising between solid grains in presence of liquid. These forces contribute strongly to the formation, deformation and flow of granular materials and they impart to them an apparent macroscopic strength (sand castle effect), even in the absence of the intrinsic cohesion or confining stress (see e.g. [1-5]). Liquid in granular materials is distributed in several ways, depending on material saturation. Starting

B. Mielniczuk

Boleslaw.Mielniczuk@umontpellier.fr

O. Millet

Olivier.Millet@univ-lr.fr

1 LaSIE, UMR CNRS 7356, University of La Rochelle, 17042 La Rochelle Cedex 1, France

2 LMGC UMR CNRS 5508, University of Montpellier, 34095 Montpellier Cedex 5, France

3 MIST, University of Montpellier, CNRS, IRSN, Montpellier, France from dry granular material, with increasing water content it pass by pendular regime, when isolated liquid bridge are observed, through funicular (capillary) regime (coalescence on isolated bridges) to full saturation (see e.g. [6,7]).

One of the most common and simple geometries used in analysis of capillary interactions is a single pendular bridge, liquid link between two solid particles. It is usually a starting point for further studies, as it constitutes also the elementary form of liquid in unsaturated granular materials (at small water content). The characteristics of pendular bridges have been analyzed for over hundred years from theoretical and experimental point of view (see e.g. [8-14]), assuming usually its circular (toroidal) shape [15-18].

In this paper, we propose several new elements concerning the analysis of mechanical interactions of capillary bridges. We use the solution of Young-Laplace equation for imposed volume, when the capillary pressure is unknown, resolved as an inverse problem with three given boundary conditions ${ }^{1}$ for second order differential equation. Subsequently, we use image treatment techniques to determine the exact profile of capillary bridge (nodoid, unduloid) and to calculate capillary force and internal pressure. The analytical model was devel-

\footnotetext{
${ }^{1}$ Filling angle $\delta$, wetting angle $\theta$ and gorge radius $y^{*}$.
} 
oped in $[14,19]$ and it is recalled in Sect. 2. In this model, profiles of pendular capillary bridges are approximated as a surface of revolution with constant mean curvature (Delaunay roulettes). Liquid bridges between two equal spheres are analyzed for fixed volumes of liquid and varying separation distance $D$ between the grains, from contact to the rupture. Main geometrical parameters (gorge radius $y^{*}$, contact angle $\theta$ and half-filling angle $\delta$ ) were determined during experimental tests, realized in laboratory and in micro-gravity conditions, using the setup described in Sect. 3. Capillary bridge were approximated as Delaunay roulettes and mean bridge curvature $H$, Laplace pressure $\Delta p$, liquid volume $V$ and capillary force $F_{c a p}$ were calculated. Results of realized tests compared with results of modeling and results of earlier experimental tests [20-23] are presented in Sect. 4 and concluded in Sect. 5. The model of capillary interfaces which we use here is based on Laplace law for pressure jumps. Possible generalization could involve a more detailed modeling of the interfacial zone, following the methods used in [24]. The results obtained allow for a more detailed description of capillary bridges in granular media: they can be used as a basis for further and promising developments the micro/macro identification process studied in [25] or to calculate the contribution of capillary stress to the total stress in unsaturated granular media [26].

\section{Theoretical framework}

We recall briefly the main results on capillary bridge properties obtained from analytical calculations of Young-Laplace equation (more details are presented in [19]). In proposed model, the knowledge of 3 input parameters $(\theta, \delta$ and $y^{*}$ ) enables to determine completely (and analytically) the meridian of the profile, the surface, volume, and associated capillary force of the capillary bridge. Seven shapes of the meridian, with constant mean curvature, are possible according to half-filling and contact angles $\delta$ and $\theta$, and to the radius of the capillary bridge neck $y^{*}$ [19]. The capillary pressure at the right hand side of Young-Laplace equation is reconstituted from the given data $\left(y^{*}, \delta, \theta\right)$ according to the following procedure.

Let us start again from the Young-Laplace equation which can be written as

$$
\frac{y^{\prime \prime}}{\left(1+y^{\prime 2}\right)^{3 / 2}}-\frac{1}{y \sqrt{1+y^{\prime 2}}}=-\frac{\Delta p}{\gamma}=: H
$$

where $\Delta p$ is a pressure difference between inside and outside of the capillary bridge, $\gamma$ is a surface tension, and $y(x)$ is the shape of the meridian of the capillary bridge. The integration of Young-Laplace equation leads to the non-linear first order differential equation [19]

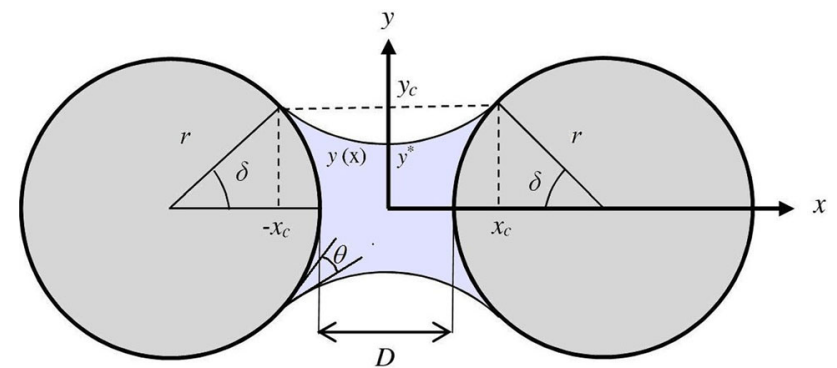

Fig. 1 Convex profile of a capillary bridge

$1+y^{\prime 2}=\frac{4 y^{2}}{H^{2}\left(y^{2}-\frac{2 \lambda}{H}\right)^{2}}$

where

$\lambda=\frac{y}{\sqrt{1+y^{\prime 2}}}+\frac{H y^{2}}{2}$

is a first integral of Young-Laplace equation. The associated capillary force may be calculated at the neck radius $y^{*}$ as:

$F_{c a p}=F_{\Delta p}+F_{S T}$

where

$F_{\Delta p}=\pi \gamma H y^{* 2}$

and

$F_{S T}=2 \pi \gamma y^{*}$

According to the signs of $H$ and $\lambda$, Eq. (2) corresponds to one that governs the Delaunay roulettes [27]

$1+y^{\prime 2}=\frac{4 a^{2} y^{2}}{\left(y^{2}+\epsilon b^{2}\right)^{2}}$

The case $\epsilon=1$ corresponds to a portion of unduloid whereas the case $\epsilon=-1$ corresponds to a portion of nodoid.

In this article, only pendular bridges between two spherical grains of radius $r$ are examined. We limit our study to concave capillary bridge with convex meridian satisfying $y^{*} \leq r \sin \delta$ (Fig. 1).

Moreover, we only focus on nodoid and unduloid capillary bridges which correspond to the main stable cases observed experimentally. The criterion of determination of the shape is recalled in the results 1 and 2, that are detailed in Appendix and can be found also in [19]. 
Once the meridian $y(x)$ is known, the volume of the liquid bridge can be calculated by

$V=\pi \int_{-x_{c}}^{x_{c}} y^{2}(x) \mathrm{d} x-2 V_{c}$

where $\pm x_{c}$ are the abscissas of triple points, $V_{c}$ is the volume of the spherical caps wet by the liquid

$V_{c}=\frac{\pi}{3} r^{3}(1-\cos \delta)^{2}(2+\cos \delta)$

If the meridian is give in a parametric form as, for example, Eqs. (13) or (17), then Eq. (5a) becomes

$V=2 \pi \frac{b^{4}}{a} \int_{0}^{\tau} \frac{(e-\cos t) \cos t \mathrm{~d} t}{(e+\cos t)^{2} \sqrt{e^{2}-\cos ^{2} t}}-2 V_{c}$

in the case of a nodoid, using Eq. (13). Similarly, for the unduloid (Eq. 17), one obtains

$V=2 \pi \frac{b^{4}}{a} \int_{0}^{\tau^{\prime}} \frac{(1-e \cos t) \mathrm{d} t}{(1+e \cos t)^{2} \sqrt{1-e^{2} \cos ^{2} t}}-2 V_{c}$

\section{Experimental procedure}

The experiment addresses the series of tests on capillary bridges between two spherical grains, with fixed water volume $V$ and varying intergranular separation $D$. Experimental setup is similar to one described in $[21,23,28]$. Monodispersed borosilicate glass spherical grains (precision of diameter $\pm 2.5 \mu \mathrm{m}$, precision of sphericity $\pm 0.625 \mu \mathrm{m}$ ), with diameter of 8 or $10 \mathrm{~mm}$ are used. Both beads are fixed to aluminum supports and positioned vertically one over with use of positioning system. Before each test sequence, beads are cleaned using $99 \%$ ethanol and left for several minutes to totally evaporate. Separation distance $D$ between the grains is controlled using a micrometer stage. Initial $D$ is set to zero (grains in contact). Then, the water drop of the given volume (from 1 to $10 \mu \mathrm{l}$ ) is introduced into the gap between them using laboratory micro-syringe (precision of $0.1 \mu \mathrm{l}$ ) to form a symmetric liquid bridge. Then distance $D$ is increasing by steps of $0.1 \mathrm{~mm}$, until the bridge is broken down. At each distance, after short time needed for bridge stabilization $(10-15 \mathrm{~s})$, the photo of the created capillary bridge is taken. For micro-gravity tests, experimental protocol is slightly different, because of time limit of $22 \mathrm{~s}$ for each zero gravity phase during parabolic flight. Beads are initially in contact, then $D$ is changed quite fast to about $1 \mathrm{~mm}$ at the beginning of zero gravity phase (for $D<1 \mathrm{~mm}$ the influence of gravity is marginal). Next, $D$ is increased gradually up to the rupture, with short stabilization time (about $3 \mathrm{~s}$ ) after each $0.2 \mathrm{~mm}$.

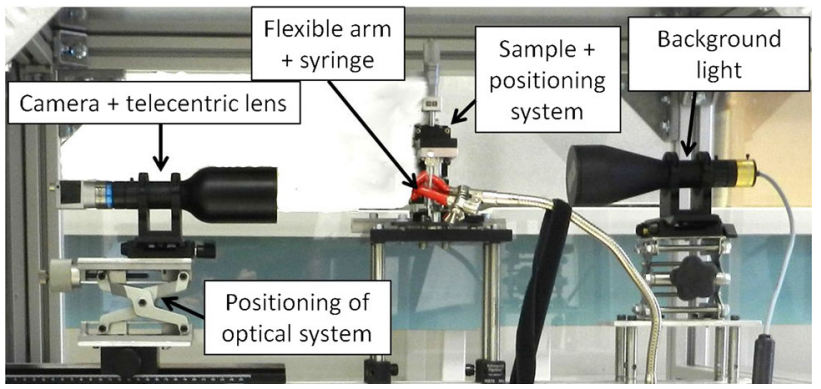

Fig. 2 Global view of experimental apparatus

Scheme of experimental configuration is presented in Fig. 2. During laboratory tests, a photo camera Nikon D5300 (resolution of 24 Mpixels) with macro-lens is used, while scientific photo camera Basler ACE (15 Mpixels) with telecentric lens was used for micro-gravity tests. To obtain the best possible contrast between capillary bridge profile and background, LED-type back-light was applied. The images are recorded using dedicated software, which allows to visualize the image in real time, to adjust camera parameters and to take photos without physical contact with the camera. Original photos are then adjusted and treated as described below. To calculate physical variables, all geometrical parameters are converted from pixels to millimeters, with use of calibration slide, with the resolution of $0.01 \mathrm{~mm}$. For each examined $V$, the sequence of 20-50 photos is obtained (changing $D$, one photo for each $D$ ). Image are adjusted with the help of image treatment tool imageJ. The part of the photo is cropped, image is binarized (using threshold option) and edges of grains and bridge are detected. The final image is represented as a table of 1 for contour and 0 otherwise. Such image is treated with Matlab code.

Main geometrical parameters of capillary bridge are presented in Fig. 3. Basing on prepared images, we define a domain within which the capillary bridge is situated, limited by $x_{u p}$ and $x_{\text {low }}$ coordinates. Next, the centers $c_{1}=$ $\left(x_{c_{1}}, y_{c_{1}}\right), c_{2}=\left(x_{c_{2}}, y_{c_{2}}\right)$, mean radius $r$ of both spheres and the coordinates of triple points $p_{1}$ to $p_{4}$ are determined. The neck radius $y^{*}$ is calculated as a half of minimal distance between the both capillary bridge meridians, corresponding to the position $x_{n k}$ on $x$-axis. Hence, the half-filling angles $\delta_{j}$ (all chosen positive) are found for each triple point $p_{j}$ by

$\delta_{j}= \begin{cases} \pm \arctan \left(\frac{y_{p_{j}}-y_{c_{1}}}{x_{p_{j}}-x_{c_{1}}}\right), & j=1,2 \\ \pm \arctan \left(\frac{y_{p_{j}}-y_{c_{2}}}{x_{p_{j}}-x_{c_{2}}}\right), & j=3,4\end{cases}$

The resulting half-filling angle $\delta$ is found as an average over all $\delta_{j}$.

The contact angle $\theta$ is calculated with use of the tangent vectors to the sphere $\mathbf{t}_{p}^{(j)}$ and to the capillary bridge profiles 


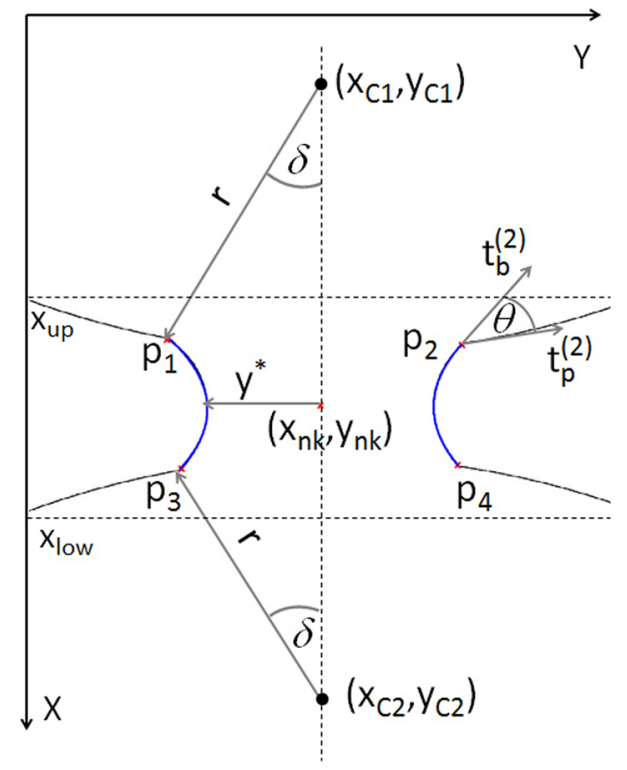

Fig. 3 Process of the image analysis used for the detection of the capillary bridge characteristics

(approximated by a sixth degree polynomials) $\mathbf{t}_{b}^{(j)}$ at the triple point $j$, pointing outside the bridge (Fig. 3). Then, the contact angles for each triple point are found as

$\theta_{j}=\arccos \left(\mathbf{t}_{b}^{(j)} \cdot \mathbf{t}_{p}^{(j)}\right), \quad j=1, \ldots, 4$

The resulting contact angle $\theta$ is taken as an average over all $\theta_{j}$. More details of image treatment procedure may be found in [29].

With the determined geometrical parameters $\left(y^{*}, \delta, \theta\right)$, the criterion for determining the shape of capillary bridge (given in Results 1 and 2) is checked and the corresponding parameters $a$ and $b^{2}$ are calculated. Then, the resulting parameterized curves (portion of nodoid or of unduloid) are plotted on the image of the capillary bridge profile obtained experimentally, as presented in Figs. 8 and 14. Variables related with capillary bridge properties (intergranular force $F_{c a p}$, Laplace pressure $\Delta p$, bridge volume $V$ and other parameters) are then calculated using equations presented in Sect. 2 and in "Appendix".

\section{Experimental results}

Using the procedure described above, experimental data are analyzed for each examined configuration. Measured geometrical parameters are used to determine the type of curve (portion of nodoid or unduloid), to reconstruct and trace the bridge profile and to calculate bridge characteristics.

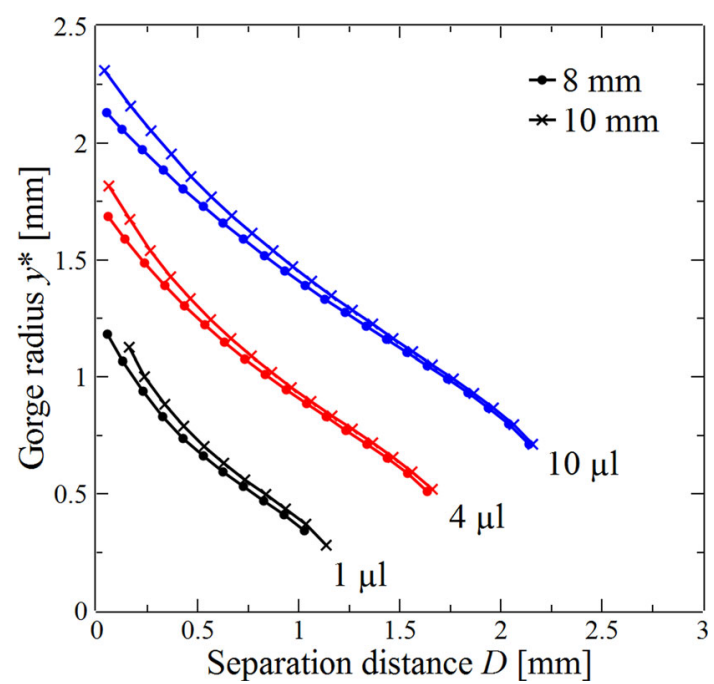

Fig. 4 The capillary bridge gorge radii $y^{*}$ as function of interparticle distance $D$ for the capillary bridge between spheres of 8 and $10 \mathrm{~mm}$

\subsection{Geometrical properties}

Evolution of geometrical parameters is presented as a function of interparticle distance $D$ in Fig. 4 (gorge radius $y^{*}$ ), Fig. 5 (mean profile curvature $H$ ) and Figs. 6 and 7 (contact angle $\theta$ and half-filling angle $\delta$ ).

Gorge radius $y^{*}$ decreases almost linearly with increasing separation distance $D$, with similar evolution for both spheres diameters. At small $D, y^{*}$ is slightly larger for $10 \mathrm{~mm}$ spheres, but $y^{*}$ at the rupture (critical gorge radius, at $D_{r}$ ) has almost the same values for both sphere diameters and it depends only on the volume of the bridge. The higher is the volume $V$, the higher separation distance at rupture $\left(D_{r}\right)$ is observed. $D_{r}$ varies from about $1.2 \mathrm{~mm}$ for $V=1 \mu 1$, to about $2.2 \mathrm{~mm}$ for $V=10 \mu 1$.

The mean curvature $H$ of the profile (Fig. 5) depends on volume of water $V$ and on separation distance $D$. Evolution of $H$ for the spheres of $10 \mathrm{~mm}$ follows the evolution of $H$ for $8 \mathrm{~mm}$ spheres, with higher $H$ for the larger beads. $H$ is initially positive and it decreases almost linearly with increase of $D$. For small volumes, the slope of the curve $H(V)$ is higher than for larger ones. For each observed case, curve $H$ passes through zero and becomes negative before the rupture of the bridge: at about $70-90 \%$ of the final separation $D$, depending on water volume $V$ (see Fig. 5). The sign of $H$ determines the sign of Laplace pressure $\Delta p$ inside the bridge (see Eq. 2) and the type of Delaunay's roulette approximating the bridge profile (see Sect. 2).

Two angles need to be determined to calculate bridge profile and physical variables: half-filling angle $\delta$ and contact angle $\theta$ (see Sect. 3). Their evolution is presented in Figs. 6 and 7. Evolution of both angles is very similar for both sphere diameters. Value of $\theta$ initially decreases with increasing $D$, 


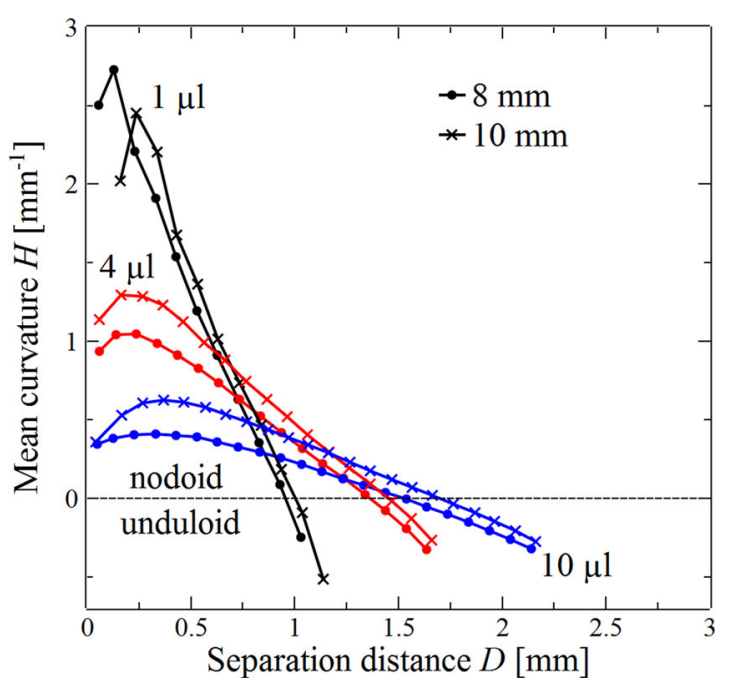

Fig. 5 The mean curvature $\mathrm{H}$ of capillary bridge between the spheres of 8 and $10 \mathrm{~mm}$, as a function of interparticle distance $D . H>0$ corresponds to the nodoid case and $H<0$ to the unduloid one

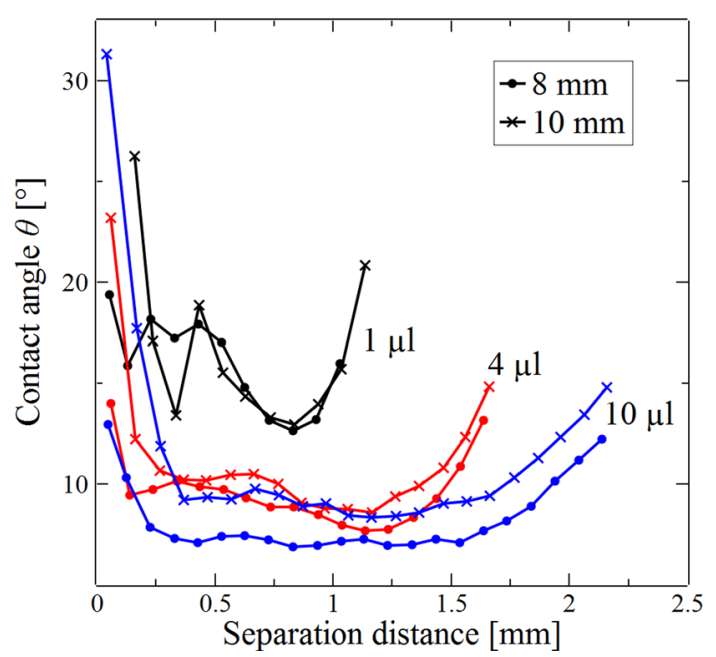

Fig. 6 Evolution of contact angle $\delta$ as a function of interparticle distance $D$ for the capillary bridge between spheres of 8 and $10 \mathrm{~mm}$

but it becomes constant very soon (not at the smallest volumes, where it fluctuates). At this moment, half-filling angle $\delta$ is still decreasing. Angle $\delta$ decreases from the beginning to about $50 \%$ of final separation $D_{r}$, than it remains constant up to the rupture. Constant value of $\delta$ denotes the pinning of contact line (see i.e. [28]). From this moment, there is no further movement of contact points (triple points), and change of separation $D$ influences only on contact angle $\theta$, which increase up to the rupture. Such phenomena was observed earlier also for evaporation sessile drops [30] and for evaporating capillary bridges [28].

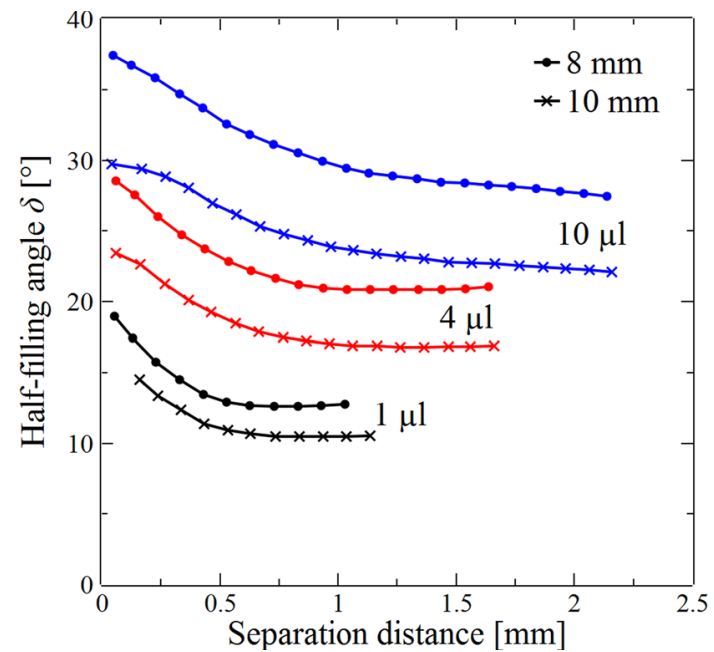

Fig. 7 Evolution of half-filling angle $\delta$ as a function of interparticle distance $D$ for the capillary bridge between spheres of 8 and $10 \mathrm{~mm}$

\subsection{Capillary bridge profiles}

The capillary bridge profile is classified as a type of Delaunay's roulette, using the criterion (12) or (16). Two types of curves were recorded: nodoid (for positive $H$ ) and unduloid (negative $H$ ). For $H=0$, catenoid shape should appear, but this profile is not stable and it has not been observed in our tests. The parameters $a$ and $b^{2}$ are found according to Eq. (14) for nodoids and Eq. (18) for unduloids. Calculated bridge profiles are superposed in the original photos to validate the approach, used in theoretical model. Example of sequence, obtained for capillary bridge between $10 \mathrm{~mm}$ spheres, with $4 \mu 1$ of water and different $D$ are presented in Fig. 8. Calculated contact points and bridge meridians are traced on recorded images.

Passage between nodoid and unduloid is observed at certain $D_{0}$, which corresponds to zero Laplace pressure $\Delta p$ (or $H=0$ ) and to the theoretical appearance of catenoid shape, which was not observed during our experiments (not stable form). Values of $D_{0}$ are presented as a function of water volume $V$ in Fig. 9. The curve passing by determined points is fitted with use of equations presented in the same Figure.

\subsection{Volume}

The defined volume of water $V$ is introduced between the grains with use of laboratory syringe. Volume $V_{i n t}$ is also calculated by integration of determined profiles (Eqs. 6 and 7 ) and by using Pappus-Guldin theorem $\left(V_{G u l}\right.$ on recorded bridge profiles). Both methods of volume calculations give similar results $\left(V_{G u l} \simeq V_{i n t}\right)$, which validates theoretical approach. The volume of the bridge is considered as 


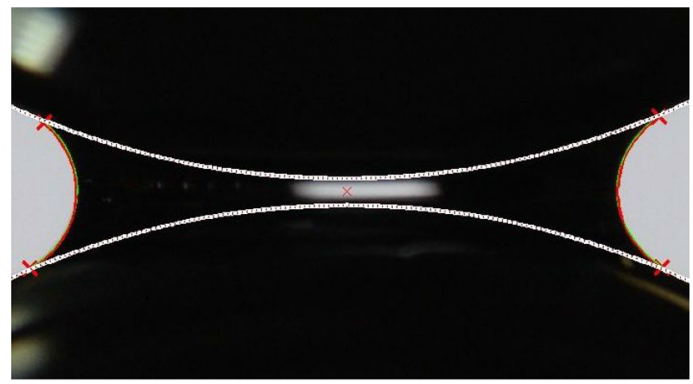

$D=0.16 \mathrm{~mm}, y^{*}=1.67 \mathrm{~mm}, \delta=22.6^{\circ}, \theta=12.2^{\circ}$, nodoid

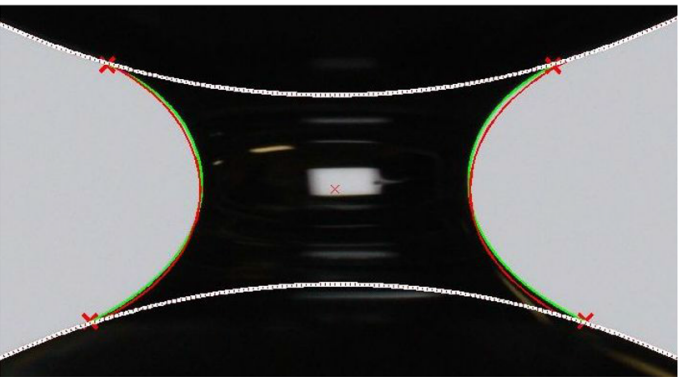

$D=1.16 \mathrm{~mm}, y^{*}=0.83 \mathrm{~mm}, \delta=16.9^{\circ}, \theta=8.6^{\circ}$, nodoid

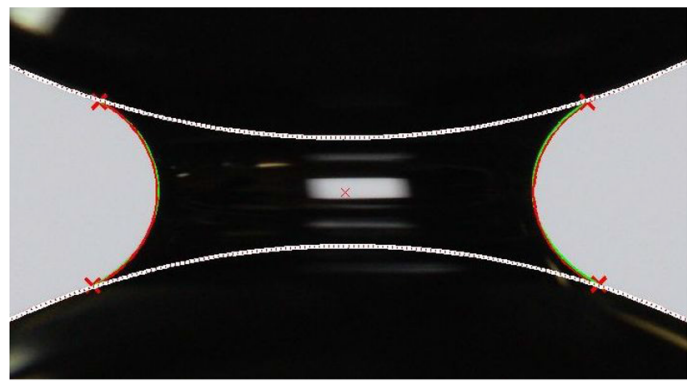

$D=0.66 \mathrm{~mm}, y^{*}=1.16 \mathrm{~mm}, \delta=17.85^{\circ}, \theta=10.5^{\circ}$, nodoid

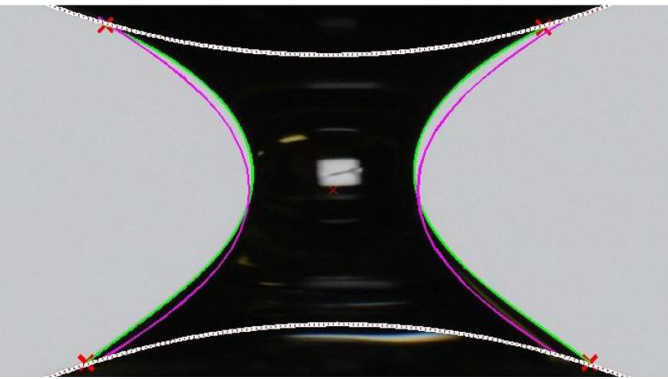

$D=1.66 \mathrm{~mm}, y^{*}=0.52 \mathrm{~mm}, \delta=16.9^{\circ}, \theta=14.8^{\circ}$, unduloid

Fig. 8 Resulting bridge profiles superposed on the original images for glass spheres of $10 \mathrm{~mm}$. The data are for fixed volume $V=4 \mu 1$ and various distance $D$ between particles (red: nodoid shape, blue: unduloid shape, calculated from theory)

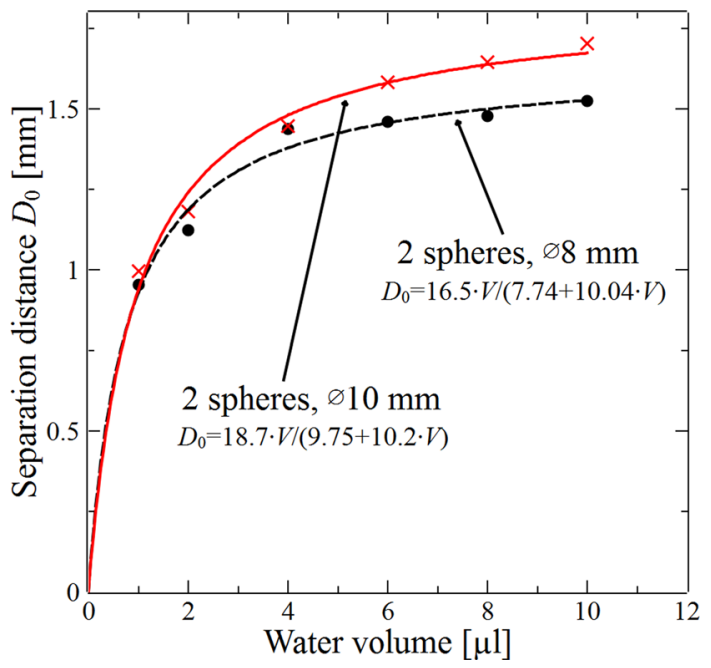

Fig. 9 Separation distance $D_{0}$ corresponding to $\Delta p=0$ and to passage from nodoid to undoloid for examined capillary bridges

constant during each test, but results presented in Fig. 10 show a decrease of $V$ during experiments (in laboratory). Although each test lasts no more than $3 \mathrm{~min}$, the volume of the bridge decrease up to $10 \%$ for the highest examined volumes, because of water evaporation from the bridge surface. Evaporation of capillary bridge will be reduced in further experiments by using a glove box with controlled atmosphere.

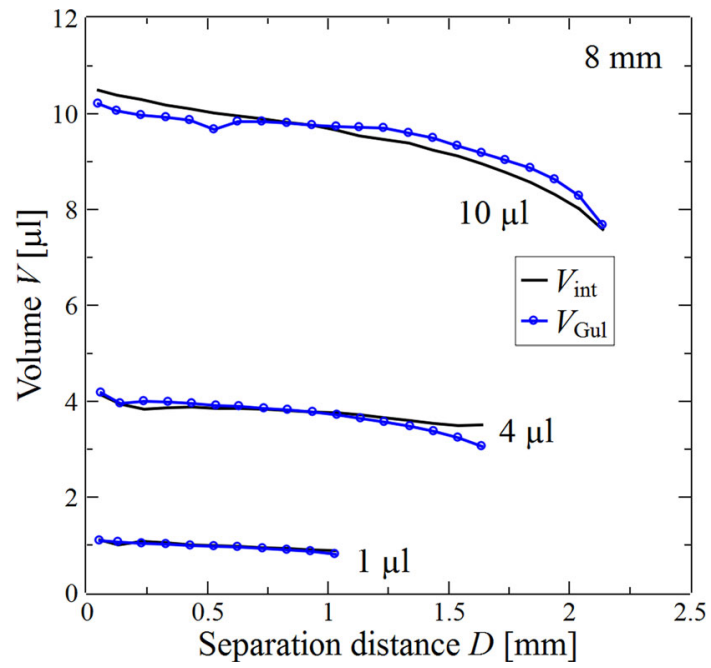

Fig. 10 The calculated capillary bridge volumes $V_{\text {int }}$ (Eqs. 6 and 7) and $V_{G u l}$ (calculated using Pappus-Guldin theorem) as a function of interparticle distance $D$ for the spheres of $8 \mathrm{~mm}$

\subsection{Laplace pressure}

Laplace pressure $\Delta p$ is proportional to the mean bridge curvature $H$ and it is calculated with use of Eq. (1) and represented in Fig. 11. Surface tension coefficient of distilled water used in experiments is $\gamma=0.072 \mathrm{~N} / \mathrm{m}$. Laplace pressure evolution follows the evolution of $H$. At the beginning $\Delta p$ is negative (positive suction), with attractive pressure resulting force $F_{\Delta p}$ (first term of Eq. (3)) acting on the surface area of 


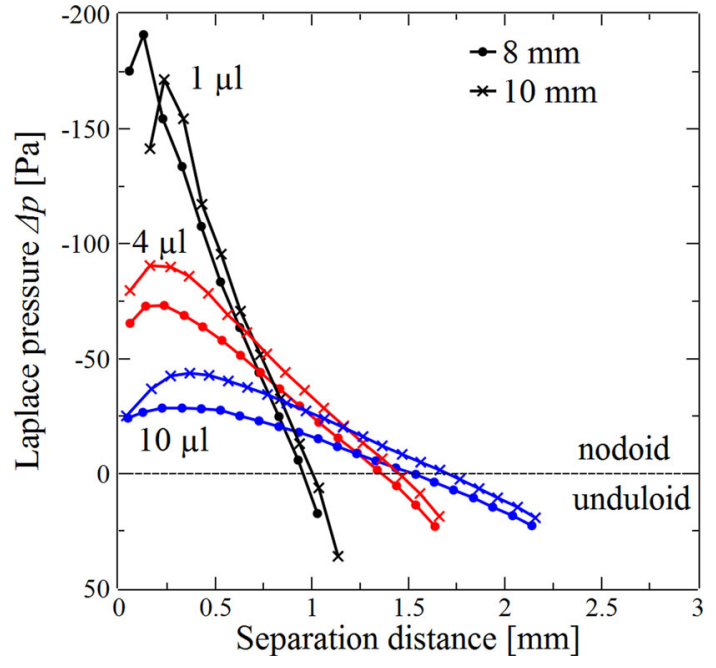

Fig. 11 The calculated Laplace pressure $\Delta p$ as a function of interparticle distance $D$ for capillary bridges between spheres of 8 and $10 \mathrm{~mm}$

capillary bridge. When curvature $H$ becomes negative, $\Delta p$ becomes positive and force $F_{\Delta p}$ becomes repulsive. Change of sign of $\Delta p$ at $D_{0}$ (Fig. 9) is linked directly to the change of geometry describing the bridge, from nodoid to unduloid shape, as presented in Fig. 8.

\subsection{Capillary force}

The capillary force $F_{c a p}$ can be evaluated by Eq. 3, including two contributing forces: pressure resulting force $F_{\Delta p}=$ $\pi \gamma H y^{* 2}$ and surface tension resulting force $F_{S T}=2 \pi \gamma y^{*}$. In Fig. 12, $F_{\text {cap }}$ is plotted as a function of the distance $D$ between the grains. In general, the decrease of $F_{c a p}$ is observed with increasing $D$. Only for $10 \mathrm{~mm}$ spheres at $10 \mu \mathrm{l}$, initial increase of $F_{c a p}$ is noted at small $D$. The rupture of liquid bridge is observed at similar $D_{\text {rup }}$ for both examined sphere diameters. The capillary force at the moment of rupture is higher for larger $V$.

Calculated $F_{c a p}$ is compared with capillary force measured in earlier experiments with use of laboratory balance (Fig. 13), [20-23]. The liquids with different surface tension coefficients were used $(\gamma=0.072 \mathrm{~N} / \mathrm{m}$ for actual results, $\gamma=0.0496 \mathrm{~N} / \mathrm{m}$ for earlier tests $[18,22,23,28])$. Both results are thus normalized with respect to $\gamma$, to make possible direct comparison of measured and calculated values. Although, in general the results are in good accordance, it is seen that for small $D$, calculated force is higher than measured one. With increasing $D$, this difference becomes smaller and close to the rupture measured force becomes higher than the calculated one. Such difference may result from different experiment conditions. Comparison of measured and calculated capillary force during the same test will be realized in a next work.

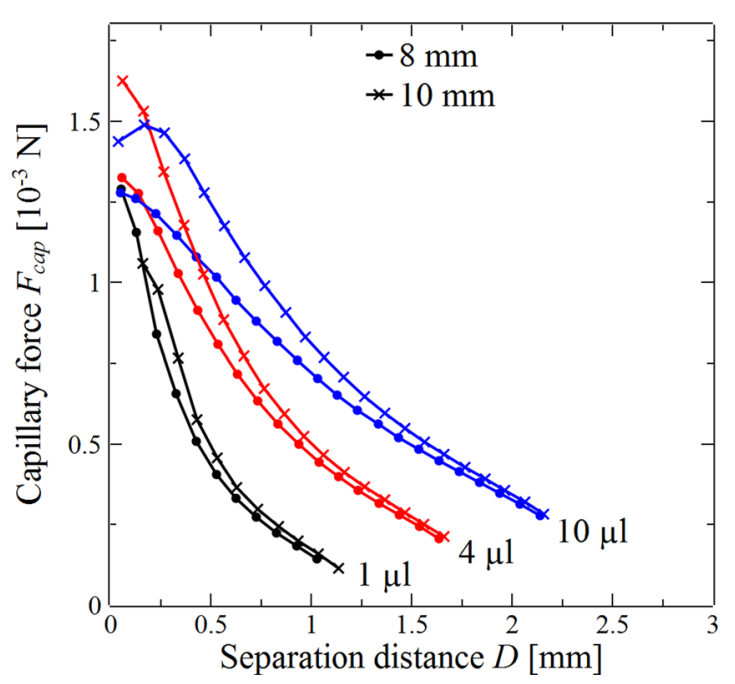

Fig. 12 Calculated capillary force $F_{c} a p$ as a function of interparticle distance $D$ for capillary bridge between spheres of 8 and $10 \mathrm{~mm}$

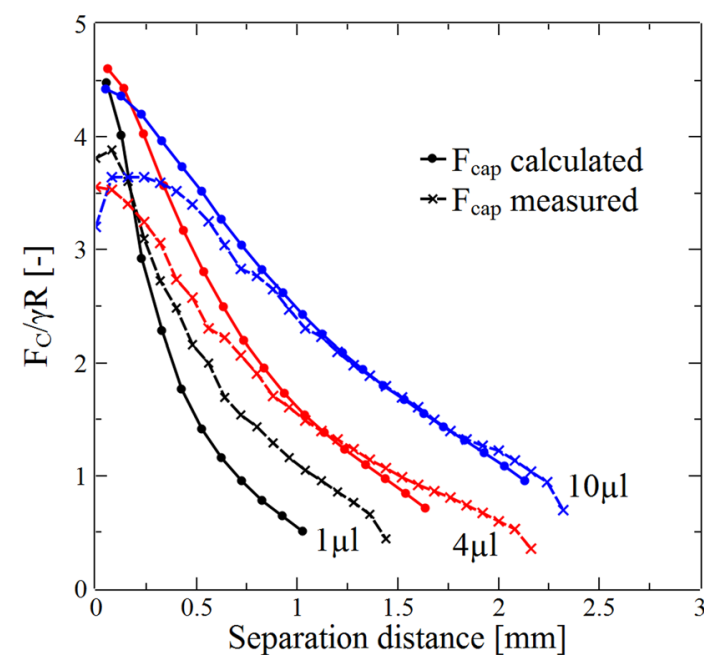

Fig. 13 Calculated and measured capillary forces $F_{\text {cap }}$ as a function of interparticle distance $D$ for the spheres of $8 \mathrm{~mm}$

\subsection{Micro-gravity tests}

For larger liquid volumes $V$ and/or a separation distance $D$ relatively high, the capillary bridge loses its symmetry due to the effects of gravity on the liquid. Water is driven towards the lower part of the bridge (see Fig. 8). To estimate the importance of the acceleration of gravity on the shape of the bridge profile, we can use the Bond number

$B o=\Delta \rho g r^{2} / \gamma$,

where $\Delta \rho$ is the difference in densities between water and air, $g$ is the acceleration of gravity. The influence of gravity is negligible if

$B o /|2 \xi r| \ll 1$

where $\xi$ is the bridge curvature [31]. 


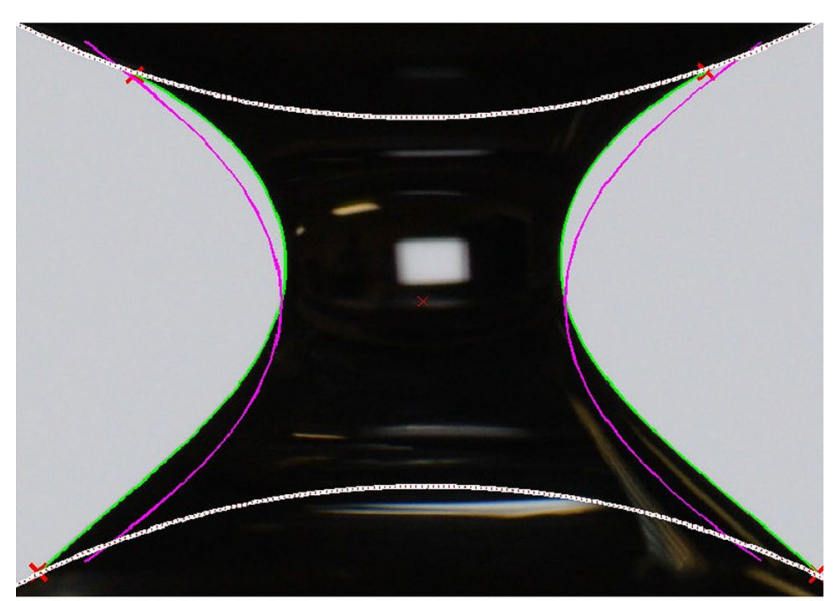

(a)

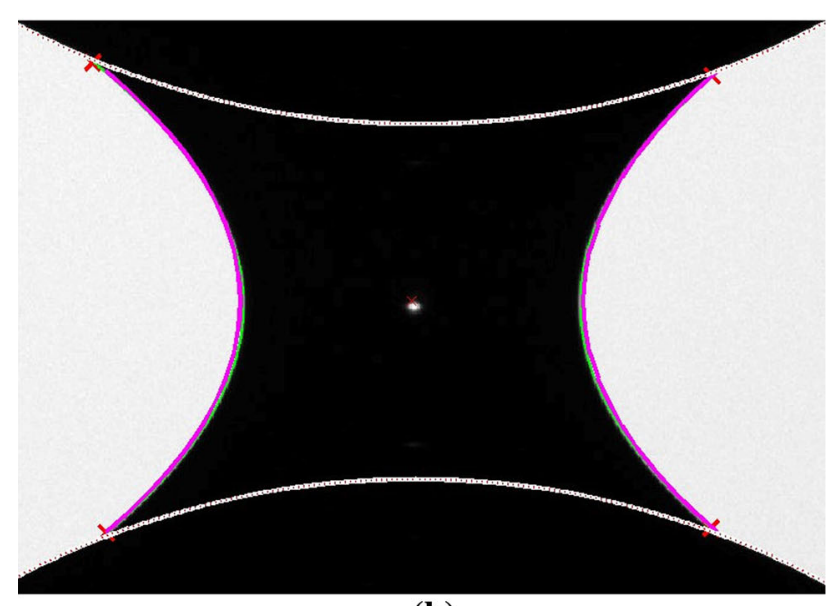

(b)

Fig. 14 Resulting bridge profiles superposed on the original images for glass spheres of $10 \mathrm{~mm}$. The data are for fixed volume $V=10 \mu 1$ and separation distance between particles $D=2.06 \mathrm{~mm}$ (blue: unduloid shape, calculated from theory). The images were recorded in laboratory (a) and during parabolic flight, in zero gravity phase (0G, b). a lab., $y^{*}=0.79 \mathrm{~mm}, \delta=22.2^{\circ}, \theta=13.5^{\circ}$, nodoid. b $0 \mathrm{G}, y^{*}=0.99 \mathrm{~mm}$, $\delta=20.8^{\circ}, \theta=20.2^{\circ}$, unduloid

This is the case for capillary bridges containing low water volume and at low separation distance. In our experiments, calculated profiles of capillary bridges fit well the ones recorded with use of photo camera, for small volumes of water and small separations. If the volume becomes higher, the gravity force may deform capillary bridge. In such cases, calculated variables are no more correct.

An example showing the influence of gravity on a capillary bridge profile is shown in Fig. 14. In this case, the value of Bond number is close to 0.3 and the influence of gravity can no longer be neglected. It can be seen that the theoretical profile, corresponding to the absence of gravity, is quite far from the actual profile of the capillary bridge. This distortion of the bridge capillary significantly alters its shape, its geometric properties and resulting capillary force.
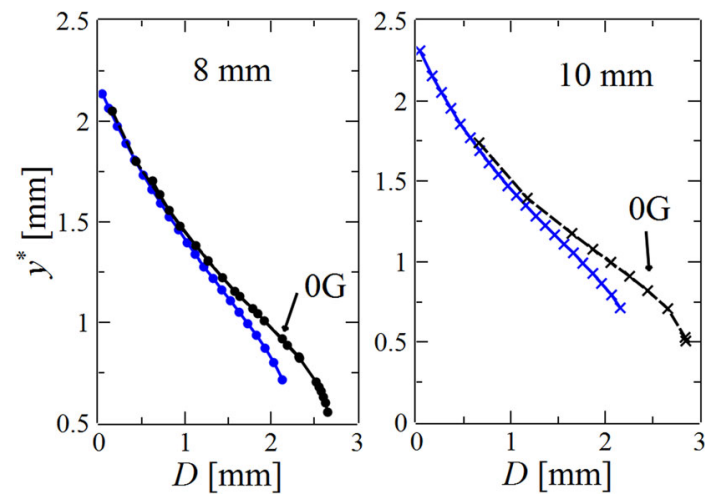

Fig. 15 Evolution of gorge radius $y^{*}$ as a function of interparticle distance $D$ for laboratory and micro-gravity tests $(0 \mathrm{G})$
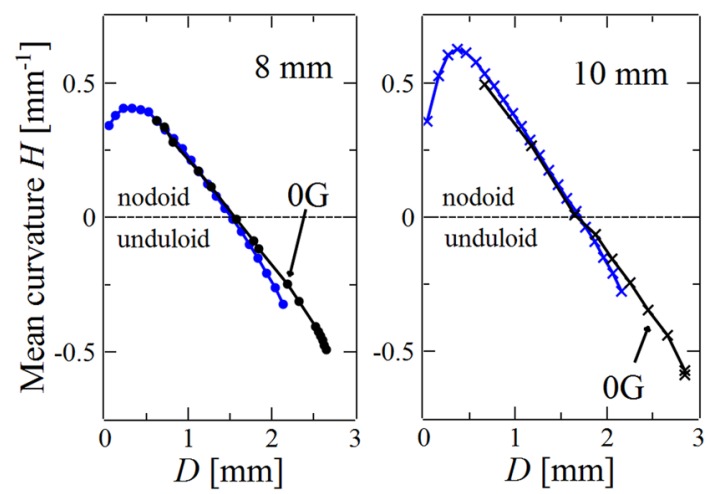

Fig. 16 Comparison of calculated mean curvature $H$ as a function of interparticle distance $D$ for laboratory and micro-gravity tests $(0 \mathrm{G})$

It has also significant consequences on the stability of the capillary bridge. For now, taking into account the gravity in the general theoretical modeling do not allow to obtain simple analytical solution. It is therefore necessary to get rid of the effects of gravity in order to validate the modeling, carried out on a wide range of parameters (volume of the liquid bridge, grain size, separation distance,...). By now, a preliminary series of tests were realized in micro-gravity conditions, during parabolic flight (joint project between LMGC, LaSIE and CNES). In Fig. 14, identical capillary bridges (diameter $=10 \mathrm{~mm}, V=10 \mu \mathrm{l}, D=2.06 \mathrm{~mm}$ ), tested in laboratory and in micro-gravity are presented, to visualize the influence of gravity on capillary bridge.

In laboratory tests, the mass of water is displaced toward the lower sphere. In consequence, gorge radius $y^{*}$ is lower (see Fig. 15), which affects on other geometrical parameters (mean curvature $H$, half-filling angle $\delta$ ) and variables (Laplace pressure $\Delta P$, capillary force $F_{c a p}$ ). Comparison of results obtained in laboratory and during parabolic flight for capillary bridges between the spheres of 8 and $10 \mathrm{~mm}$, with the volume of $10 \mu 1$, is presented in Figs. 15 (gorge radius), 16 (mean curvature) and 17 (capillary force). 

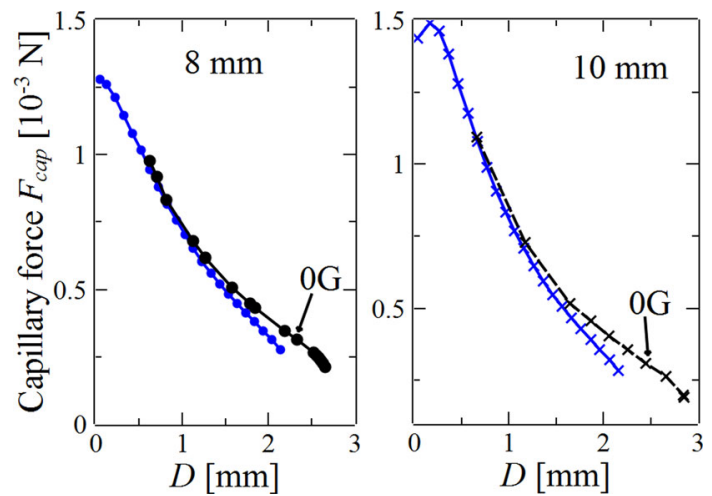

Fig. 17 Comparison of calculated capillary forces $F_{\text {cap }}$ as a function of interparticle distance $D$ for laboratory and micro-gravity tests $(0 \mathrm{G})$

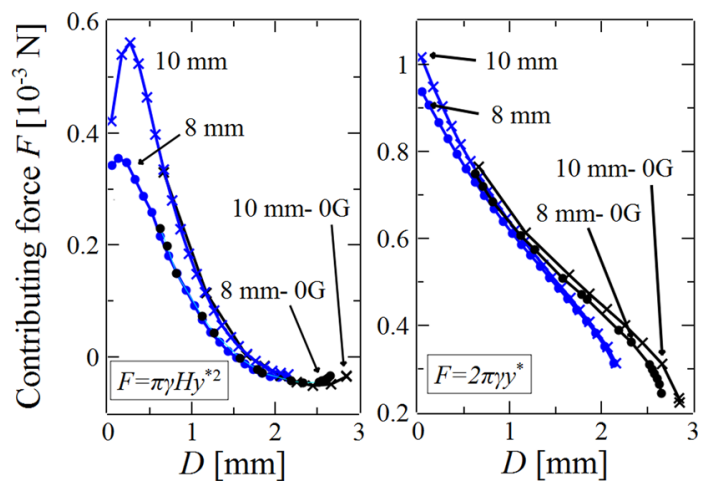

Fig. 18 Comparison of two calculated contributions to total capillary forces $F_{\text {cap }}$ (see Eq. 3) as a function of interparticle distance $D$, for laboratory and micro-gravity tests $(0 \mathrm{G})$

Is is seen, that gorge radius $y^{*}$ is higher for micro-gravity tests, particularly for higher $D$. Also other geometrical parameters $(\delta, \theta, H)$ differ significantly. In results, calculated $\Delta p$ and $F_{c a p}$ close to the rupture are larger for bridges tested in micro-gravity conditions. In addition, the rupture of capillary bridges occurs at larger separations $D$, in particular for capillary bridges between the spheres of bigger diameter. However, the passage between two types of Delanuay's roulettes occurs at the same separation distance for laboratory and micro-gravity tests (see Fig. 16). The gravity influences differently the two forces contributing to total capillary force $F_{c a p}$, as showed in Fig. 18. Almost no changes in $F_{\Delta p}$ are observed between laboratory and micro-gravity tests (maximum difference of $0.007 \cdot 10^{-3} \mathrm{~N}$ for $8 \mathrm{~mm}$ spheres and $0.013 \cdot 10^{-3} \mathrm{~N}$ for $10 \mathrm{~mm}$ spheres), while $F_{S T}$ is visibly higher for micro-gravity tests (maximum difference of 0.077 .
$10^{-3} \mathrm{~N}$ for $8 \mathrm{~mm}$ spheres and $0.086 \cdot 10^{-3} \mathrm{~N}$ for $10 \mathrm{~mm}$ spheres).

\section{Conclusions}

In this paper, knowing the geometry of the granular assembly in the pendular regime (half-filling and contact angles, gorge radius), we propose a simple way of calculating/determining the exact geometric properties of capillary doublets and the associated capillary force and Laplace pressure. The accuracy and validity of the model was demonstrated by comparison with the experimental results. The results from theoretical modeling match very accurately experimental results for small liquid volumes and small separations between particles. For larger ones, the capillary bridge loses its symmetry due the effects of gravity. In order to remove the distortion effect due to gravity, initial experiments in micro-gravity conditions, during a parabolic flight (Airbus ZeroG of Novespace society and CNES) have been performed. Realisation of the experiments in microgravity enables to separate the couplings between the gravity and capillary effects, it simpifies theoretical modeling and comparison with experiments. We found that the profile of capillary bridge profile evolves from nodoid to unduloid shape with increasing separation distance $D$ between the grains. This passage is accompanied by the increase of internal pressure, from initially negative values to positive pressure at the rupture, while capillary force decreases (with increasing $D$ ). Experimental validation of proposed model justifies its further development, with its adaptation to other configurations of capillary bridges (sphere-plate, plate-plate), including of dynamic processes (extension, evaporation, coalescence of pendular bridges) and analysis of stability/rupture of liquid bridges, envisaged in the nearest future. Enhanced analytical model will be also validated experimentally in laboratory and in micro-gravity conditions. It would also constitute a base for further modeling in macroscopic scale (sample scale).

Acknowledgements The part of the work concerning the experiments in micro-gravity was supported by the CNES (joint project PARABOLE 2015, VP118, between LaSIE-LMGC-CNES).

\section{Compliance with ethical standards}

Conflict of interest The authors declare that they have no conflict of interest. 


\section{Appendix: Results for determination of nodoid and unduloid shape}

Result 1 If the observed data $\left(y^{*}, \delta, \theta\right)$ are such that

$r \sin \delta \sin (\delta+\theta)<y^{*}<r \sin \delta$

with $H>0$ and $\lambda>0$, then the meridian of the capillary bridge is a portion of nodoid whose parameterization is given by

$x(t)=\frac{b^{2}}{a} \int_{0}^{t} \frac{\cos u \mathrm{~d} u}{(e+\cos u) \sqrt{e^{2}-\cos ^{2} u}}$

$y(t)=b \sqrt{\frac{e-\cos t}{e+\cos t}}, \quad t \in[-\tau, \tau]$

where

$\tau=\arccos \left(e \frac{b^{2}-r^{2} \sin ^{2} \delta}{b^{2}+r^{2} \sin ^{2} \delta}\right)$

$e=\frac{\sqrt{a^{2}+b^{2}}}{a}$

is the unique solution in $(0, \pi / 2)$ of the equation $y(\tau)=$ $r \sin \delta$. The associated geometrical parameters are given by:

$a=\frac{1}{2} \frac{r^{2} \sin ^{2} \delta-y^{* 2}}{y^{*}-r \sin \delta \sin (\delta+\theta)}$

$b^{2}=y^{*} r \sin \delta \frac{r \sin \delta-y^{*} \sin (\delta+\theta)}{y^{*}-r \sin \delta \sin (\delta+\theta)}$

The capillary pressure $\Delta p$ and the mean curvature $H$ may be calculated as:

$$
\begin{aligned}
\Delta p & =-2 \gamma \frac{y^{*}-r \sin \delta \sin (\delta+\theta)}{r^{2} \sin ^{2} \delta-y^{* 2}}, \\
H & =-\frac{\Delta p}{\gamma}=\frac{1}{a}
\end{aligned}
$$

Result 2 If the observed data $\left(y^{*}, \delta, \theta\right)$ are such that

$0<y^{*}<r \sin \delta \sin (\delta+\theta)$

with $H<0$ and $\lambda>0$, then the meridian of the capillary bridge is a portion of unduloid whose parameterization is given by:

$x(t)=\frac{b^{2}}{a} \int_{0}^{t} \frac{\mathrm{d} u}{(1+e \cos u) \sqrt{1-e^{2} \cos ^{2} u}}$

$y(t)=b \sqrt{\frac{1-e \cos t}{1+e \cos t}}, \quad t \in\left[-\tau^{\prime}, \tau^{\prime}\right]$

where

$\tau^{\prime}=\arccos \left(\frac{1}{e} \frac{b^{2}-r^{2} \sin ^{2} \delta}{b^{2}+r^{2} \sin ^{2} \delta}\right)$

is a unique solution in $(0, \pi / 2)$ of the equation $y\left(\tau^{\prime}\right)=$ $r \sin \delta$. The geometrical parameters are given by:

$a=\frac{1}{2} \frac{r^{2} \sin ^{2} \delta-y^{* 2}}{r \sin \delta \sin (\delta+\theta)-y^{*}}$

$b^{2}=y^{*} r \sin \delta \frac{r \sin \delta-y^{*} \sin (\delta+\theta)}{r \sin \delta \sin (\delta+\theta)-y^{*}}$

The capillary pressure $\Delta p$ and the mean curvature $H$ may be calculated as:

$\Delta p=\frac{\gamma}{a}, \quad H=-\frac{1}{a}$ 


\section{References}

1. Peron, H., Delenne, J.Y., Laloui, L., El Youssoufi, M.S.: Discrete element modelling of drying shrinkage and cracking of soils. Comput. Geotech. 36, 61 (2009)

2. Princen, H.M.: Comments on 'The effect of capillary liquid on the force of adhesion between spherical solid particles'. J. Colloid Interface Sci. 26, 249 (1968)

3. Rahardjo, H., Fredlund, D.G.: Experimental verification of the theory of consolidation for unsaturated soils. Can. Geotech. J. 32, 749 (1995)

4. El Youssoufi, M.S., Delenne, J.Y., Radjai, F.: Self-stresses and crack formation by particle swelling in cohesive granular media. Phys. Rev. E 71, 051307 (2005)

5. Richefeu, V., El Youssoufi, M.S., Radjai, F.: Shear strength properties of wet granular materials. Phys. Rev. E 73, 051304 (2006)

6. Flemmer, C.L.: On the regime boundaries of moisture in granular materials. Powder Technol. 66, 191 (1991)

7. Herminghaus, S.: Dynamics of wet granular matter. Adv. Phys. 54, $221(2005)$

8. Young, T.: An essay on the cohesion of fluids. Philos. Trans. 95, 65 (1805). https://doi.org/10.1098/rstl.1805.0005

9. Plateau, J.: The Annual Report of the Smithsonian Institution, Washington DC pp. 338-369 (1864)

10. Mason, G., Clark, W.C.: Liquid bridges between spheres. Chem. Eng. Sci. 20, 859 (1965)

11. Haines, W.B.: Studies of the physical properties of soils. II. A note on the cohesion developed by capillarity forces in an ideal soil. J. Agric. Sci. 15, 529 (1925)

12. Megias-Alguacil, D., Gauckler, L.J.: Capillary forces between two solid spheres linked by a concave liquid bridge: regions of existence and forces mapping. AiChE J. 55, 1103 (2009)

13. Rabinovich, Y.I., Esayanur, M.S., Moudgil, B.M.: Capillary forces between two spheres with a fixed volume liquid bridge: theory and experiment. Langmuir 21, 10992 (2005)

14. Gagneux, G., Millet: An analytical framework for evaluating the cohesion effects of coalescence between capillary bridges. Granular Matter 18 (2016). https://doi.org/10.1007/s10035-0160613-5

15. Lian, G., Thornton, C., Adams, M.J.: A theoretical study of the liquid bridge forces between two rigid spherical bodies. J. Colloid Interface Sci. 161, 138 (1993)

16. Willett, C.D., Adams, M.J., Johnson, S.A., Seville, J.P.K.: Capillary bridges between two spherical bodies. Langmuir 16, 9396 (2000)

17. Soulie, F., Cherblanc, F., El Youssoufi, M.S., Saix, C.: Influence of liquid bridges on the mechanical behaviour of polydisperse granular materials. Int. J. Numer. Anal. Meth. Geomech. 30, 213 (2006)

18. Gras, J.P., Delenne, J.Y., El Youssoufi, M.S.: Study of capillary interaction between two grains: a new experimental device with suction control. Granul. Matter 15, 49 (2013). https://doi.org/10. 1007/s10035-012-0388-2
19. Gagneux, G., Millet, O.: Analytic calculation of capillary bridge properties deduced as an inverse problem from experimental data. Transp. Porous Media 105, 117 (2014)

20. Hueckel, T., Mielniczuk, B., El Youssoufi, M.S.: Micro-scale study of rupture in desiccating granular media. In: Proceedings of GeoCongress 2013, Geotechnical Special Publication GSP ASCE, vol. 231, p. 808 (2013)

21. Mielniczuk, B., Hueckel, T., El Youssoufi, M.S.: Micro-scale testing of capillary bridge evolution due to evaporation. In: Laloui, L, Ferrari, A (eds.) Multiphysical Testing of Soils and Shales, Springer Series in Geomechanics and Geoengineering, pp. 233-238 (2013)

22. Mielniczuk, B., El Youssoufi, M.S., Sabatier, L., Hueckel, T.: Rupture of a liquid bridge between two grains due to its evaporation. Acta Geophys. 62, 1087 (2014). https://doi.org/10.2478/s11600014-0225-6

23. Mielniczuk, B., Hueckel, T., El Youssoufi, M.S.: Evaporationinduced evolution of the capillary force between two grains. Granul. Matter 16, 815 (2014)

24. Dell'Isola, F., Gouin, H., Rotoli, G.: Nucleation of spherical shelllike interfaces by second gradient theory: numerical simulations. Eur. J. Mech. B/Fluids 15(4), 545 (1996)

25. Madeo, A., dell'Isola, F., Darve, F.: A continuum model for deformable, second gradient porous media partially saturated with compressible fluids. J. Mech. Phys. Solids 61, 82196 (2013)

26. Duriez, J., Eghbalian, M., Wan, R., Darve, F.: The micromechanical nature of stresses in triphasic granular media with interfaces. J. Mech. Phys. Solids (2016). https://doi.org/10.1016/j.jmps.2016. 10.011

27. Delaunay, C.H.: Sur la surface de révolution dont la courbure moyenne est constante. Journal de mathématiques pures et appliquées 6, 309 (1841)

28. Mielniczuk, B., Hueckel, T., El Youssoufi, M.S.: Laplace pressure evolution and four instabilities in evaporating two-grain liquid bridges. Powder Technol. 283, 131 (2015). https://doi.org/10.1016/ j.powtec.2015.05.024

29. Gagneux, G., Millet, O., Mielniczuk, B., El Youssoufi, M.S.: Theoretical and experimental study of pendular regime in unsaturated granular media. Eur. J. Environ. Civil Eng. pp. 1-14 (2016). https:// doi.org/10.1080/19648189.2016.1167782

30. Bourges-Monnier, C., Shanahan, M.E.R.: Influence of evaporation on contact angle. Langmuir 11, 2820 (1995). https://doi.org/10. 1021/la00007a076

31. Adams, M.J., Johnson, S.A., Seville, J.P.K., Willett, C.: Mapping the influence of gravity on pendular liquid bridges between rigid spheres. Langmuir 18, 6180 (2002) 